\title{
CYTOSKELETON IN REGENERATING PROTOPLASTS AND RESTORATION OF CELL POLARITY IN THE YEAST SACCHAROMYCES CEREVISIAE*
}

\author{
A. Svoboda, ${ }^{* *}$ Iva Slaninová and Alena HolubáŘová \\ Department of Biology, Medical Faculty, Masaryk University, \\ Joštova 10, 66243 Brno, Czech Republic
}

(Received: August 31, 2000; accepted: October 5, 2000)

\begin{abstract}
The actin cables and microtubules were disrupted during protoplasting in Saccharomyces cerevisiae cells. In the process of protoplast regeneration, the cytoplasmic microtubules were the first to be restored; the actin patches remained regularly distributed under the surface of the growing protoplast. After the cell wall had been regenerated in a gelatine medium, the actin patches aggregated into clusters, which marked the site of bud development. An incomplete cell wall was the apparent cause for uncoupling between karyokinesis and cytokinesis. The presence of several nuclei in the cell gave rise to several buds emerging simultaneously and was probably related to their random positions. In haploids, however, the axial type of budding was also obvious in the reverting protoplasts. These observations suggest that actin is a structure involved in the restoration of polar growth during protoplast regeneration, and that the cell wall plays an important role in this process: in its absence, actin fails to polarise.
\end{abstract}

Keywords: Saccharomyces cerevisiae - yeast protoplasts - microtubules - actin - cell wall

\section{INTRODUCTION}

The understanding of gene and spatial control of cell polarity has greatly advanced in the last two decades, particularly as concerns the yeast Saccharomyces cerevisiae. Genes have been defined whose products are responsible for identification of the site of polar growth and for the assembly of molecular machinery directing actin and the secretory pathway towards this site [7]. The importance of the genes determining polarity is supported by the fact that their mutant forms result in a loss of polarity and, consequently, in spherical cell growth $[1,16]$.

A structure vitally important for polar growth, both in budding and mating, is actin, which is present as cables and patches [7], each form having different functions in cell polarity control $[21,22]$. The cytoplasmic or astral microtubules are primarily responsible for the orientation and movement of the nucleus in relation to the cell axis, while the spindle microtubules are involved in chromosome separation and nuclear division $[7,25]$.

\footnotetext{
*Dedicated to Professor Lajos Ferenczy on the occasion of his 70th birthday.

**Corresponding author; e-mail: asvoboda@med.muni.cz
} 
Most of the studies on cell polarity control have been based on the regular cell morphology. Protoplasts, spherical bodies which lack cell walls, can be regarded as non-polar forms. They are able to regenerate their cell walls and to revert to normal cells [14]. Although protoplasts are capable of polar growth, this polarity is rather passive and depends on the properties of a new, incompletely regenerated wall [14]. The recovery of species-specific cell morphogenesis is not a one-step process, but usually requires several repair attempts [13]. In our laboratory, we have repeatedly demonstrated that the presence of the cell wall is a decisive factor in the restoration of normal cell morphogenesis $[2,13,18]$.

However, protoplasts as models for studies on cell polarity have been neglected in recent years. There have been only a few reports in which protoplasts have been used to investigate the morphogenesis of yeast cells $[2,9,10,17]$.

The results presented here show that the protoplast state of a cell results in disruption of the microtubular and actin cytoskeletons and in a disturbance of cell polarity. The microtubular skeleton is repaired during the process of cell wall regeneration and, after this has been completed, the actin cytoskeleton and the polar organisation of the cell are restored. This implies that the cell wall is involved in the control of cell polarity and also plays a role in the functioning of a molecular complex that initiates polar growth.

\section{MATERIALS AND METHODS}

Organisms and culture conditions: Saccharomyces cerevisiae, strain CCY 21-4-59, prototrophic tetraploid, and prototrophic diploid strain $71 \alpha \alpha$, derived from the tetraploid. The strains were maintained on malt extract agar. Cells were grown in liquid malt extract medium at $29^{\circ} \mathrm{C}$.

Protoplast preparation and cultivation: Protoplasts were prepared from cells during the logarithmic phase of growth by means of NovoZym 234 (Novo Industri A/B, Nagsvaerd, Denmark; $1 \mathrm{mg} / \mathrm{mL}$ ) in $0.7 \mathrm{M}$ non-buffered mannitol or $0.6 \mathrm{M} \mathrm{KCl}$. After washing, protoplasts were suspended in MEM (malt extract medium containing 0.7 M mannitol) or in YNBG medium $(0.67 \%$ (w/v) Yeast Nitrogen Base Difco; $2 \%$ (w/v) glucose; $0.7 \mathrm{M}$ mannitol; $\mathrm{pH}$ 5.4). For reversion experiments, protoplasts were embedded in $30 \%(\mathrm{w} / \mathrm{v})$ Difco gelatine in YNBG at $38^{\circ} \mathrm{C}$ and cultivated at laboratory temperature $\left(22-24{ }^{\circ} \mathrm{C}\right)$. The final concentration of protoplasts in gelatine medium ranged from $10^{5}$ to $10^{6}$ per $\mathrm{mL}$.

Fixation and permeabilisation of protoplasts: A culture of protoplasts in liquid YNBG medium was mixed at a ratio of $3: 1$ with a fixative containing $15 \%$ formaldehyde, $0.5 \mathrm{mM} \mathrm{MgCl}$ and $1 \mathrm{mM}$ EGTA in $100 \mathrm{mM}$ potassium phosphate buffer at $\mathrm{pH} 6.5[4,20,24]$ and allowed to stand for $90 \mathrm{~min}$ at room temperature. Samples were washed with the buffer and permeabilised with $1 \%$ Triton X 100 for $20 \mathrm{~min}$.

The samples of protoplasts grown in gelatine medium were overlayered with 10 volumes of liquid YNBG medium and incubated at $37^{\circ} \mathrm{C}$ for $10 \mathrm{~min}$. Each sample 
was first carefully shaken and then centrifuged; the pellet was washed twice with YNBG medium at $37^{\circ} \mathrm{C}$. Before fixation the samples were incubated at laboratory temperature for $30 \mathrm{~min}$. If needed, the fixed regenerated protoplasts were treated with NovoZym $234(1 \mathrm{mg} / \mathrm{mL})$ in the buffer at room temperature for $15 \mathrm{~min}$.

Fluorescence microscopy: The fixed and permeabilised protoplasts were first incubated in bovine serum albumin for 20 min and then overnight in TAT1 antibody $[24,26]$. With a secondary antibody added, the suspensions were allowed to stand for $45 \mathrm{~min}$ at room temperature. After washing, the cells were transferred to a mounting medium with DAPI (4'-6-diamidino-2-phenylindole) [20] in order to stain the DNA in the nuclei and mitochondria. The actin cytoskeleton was stained with rhodaminephalloidin (Molecular Probes) according to [19].

The preparations were viewed in a Leica Laborlux fluorescence microscope.

\section{RESULTS}

\section{Actin structures and microtubules in fresh protoplasts}

When the cell wall was removed, the oval cell shape turned to spherical, the location of the organelles changed and both the actin and microtubular structures disintegrated. In fresh protoplasts, the actin cables disappeared and the actin patches were distributed subcortically along the whole inner surface (Fig. 1). The anti-tubulin antibody detected only fluorescent dots close to the nucleus; these were identified as spindle pole bodies (SPBs) (Fig. 2). In protoplasts cultivated in MEM or YNBG for 30 to $60 \mathrm{~min}$, microtubules reappeared as asters emanating from the SPBs (Fig. 3). The early renewal of cytoplasmic microtubules contrasted with the absence of spindles; these appeared later, probably as late as the next round of mitosis. The actin morphology remained unchanged: only actin patches irregularly distributed under the protoplast surface were observed.

\section{Protoplast development under conditions allowing regeneration of an incomplete cell wall}

Further cultivation in the liquid medium produced an increase in protoplast volume. The protoplasts grew polarly, appearing as a sequence of hernias. It was found earlier that their surface was covered by a fibrillar wall material [14]. The actin patches were randomly distributed under the whole surface of each protoplast (Fig. 4). Long cytoplasmic microtubules emanating from the SPBs extended along the axis of the growing protoplast (Fig. 5). Karyokinesis occurred but the intervals between the nuclear divisions grew longer: after $6 \mathrm{~h}$ of cultivation, usually 2 , but at most 4 nuclei were observed. After $2 \mathrm{~h}$ of incubation, the growing protoplasts gave rise to aggregations in which it was difficult to discern the original boundaries between the individual protoplasts. After 4 to $10 \mathrm{~h}$, the protoplasts growing in liquid media usually lysed. 


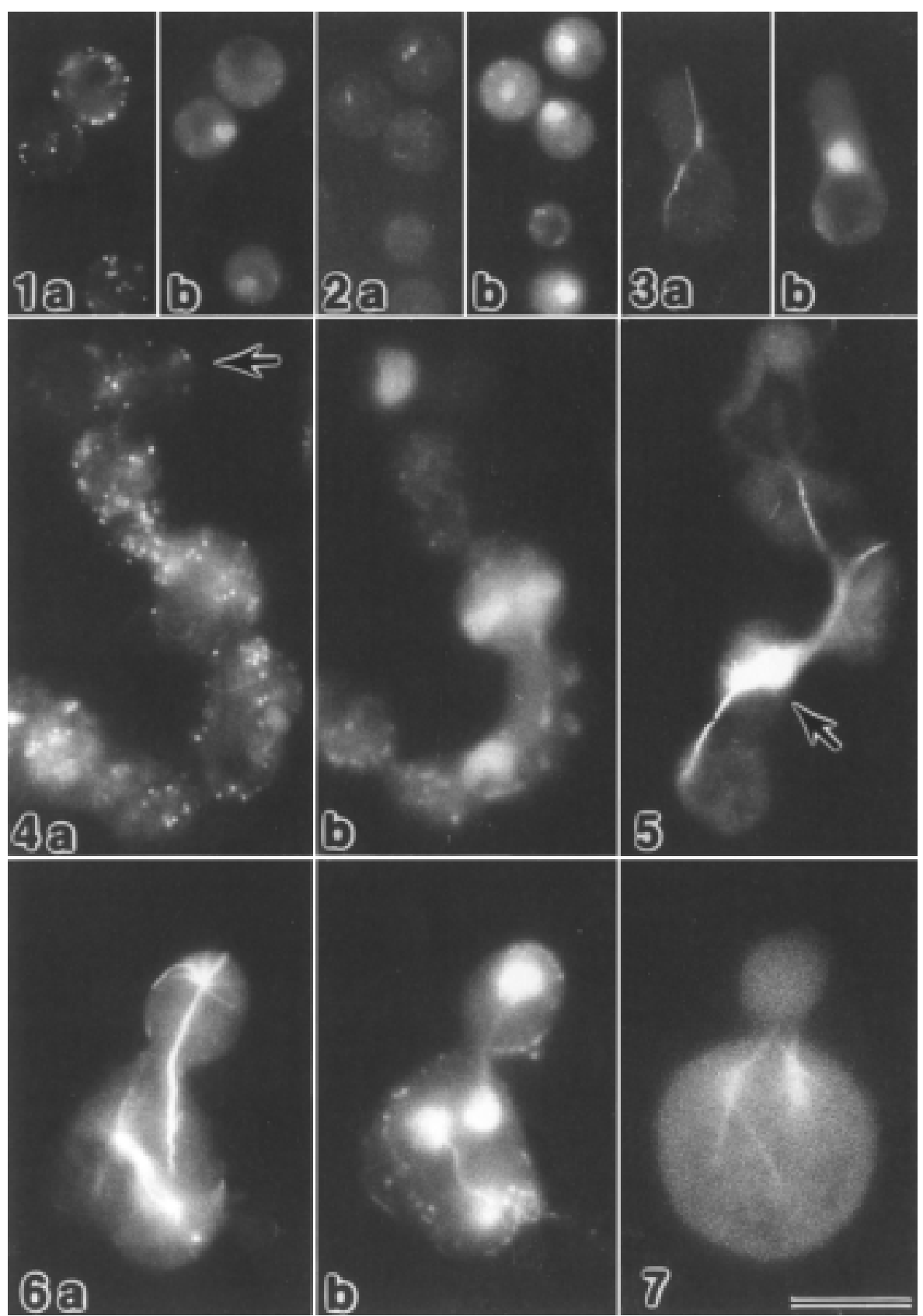

Figs 1-7. - 1. Freshly isolated protoplasts. Actin patches are seen at the periphery of protoplasts (rhodamine-phaloidin staining) (a); nuclei are stained by DAPI (b). For Figs 1-7 the bar represents $10 \mu \mathrm{m}$ - 2. Disintegrated microtubular structures after protoplasting. Fluorescent dots (a) are located at regions of nuclei (b). - 3. Restoration of microtubular cytoskeleton after incubation for $60 \mathrm{~min}$ in MEM.

Acta Biologica Hungarica 52, 2001 


\section{Protoplast development under conditions allowing regeneration of a complete cell wall}

Protoplasts embedded into an osmotically stabilised medium supplemented with $30 \%$ gelatine displayed a typical growth morphology: they grew spherically and a distinct layer of cell wall appeared on their surface. A previous study revealed [14] that gel media were necessary only for the first stage of regeneration in order to facilitate the assembly of a layer of a complete cell wall. Accordingly we studied the cytoskeletal structures in protoplasts cultivated in liquid medium after 6 to $8 \mathrm{~h}$ of preincubation in a gelatine medium. This enabled us to fix the regenerating protoplasts without additional manipulation.

In spherically growing protoplasts, the nuclei divided synchronously, but during the wall regeneration period ( 6 to $8 \mathrm{~h}$ ) they divided only once or twice; this implies that the nuclear division was retarded. Bundles of astral cytoplasmic microtubules emanated from the SPBs of each nucleus, which was either dividing or non-dividing. If a protoplast had two nuclei, these divided synchronously and were randomly located. Irregularly distributed actin patches were observed at the protoplast periphery.

During reversion, one nucleus was usually transported into the bud, leaving the parental protoplast with an odd number of nuclei. The location of the nuclei during karyokinesis varied. The spindle of one of the dividing nuclei was oriented towards the bud, as in normal cells, while the axis of the other dividing nucleus was directed away from it (Fig. 6). The bud necks were so wide that, in some of the protoplasts, the spindles of two nuclei extended into one bud (Fig. 7). This may explain the higher number of nuclei in the first generation of reverted cells.

After growing as spheres for 10 to $14 \mathrm{~h}$, the protoplasts began to bud. The revertants in the first generation were large and abnormal in form, but the cells produced in the subsequent generations reverted step by step to species-specific morphology (Fig. 8).

The actin in the spherically growing protoplasts was still present as patches randomly distributed under the whole surface. Before bud emergence, actin patches clustered at the site of a prospective bud (Fig. 9). Fine cables, which also assembled, radiated from these sites (not discernible in this micrograph). In one protoplast, two or three clusters of actin patches could be observed, which indicates the formation of several buds at the same time (Fig. 10).

Cytoplasmic microtubules emanate from the SPBs (a) of a mitotic nucleus (b). - 4. Polar growth of a protoplast after incubation for $4 \mathrm{~h}$ in MEM. Note actin patches spreading along the whole protoplast length and the absence of concentrated patches at the tip (a, arrow); four nuclei in the same protoplast (b). 5. Strong fluorescence of a spindle (arrow) and long cytoplasmic microtubules are seen in a polarly growing protoplast. -6 . Two dividing nuclei in a reverting protoplast. Note two spindles (a) and corresponding nuclei (b). -7 . Two spindles directing to one bud during budding of a regenerated protoplast 


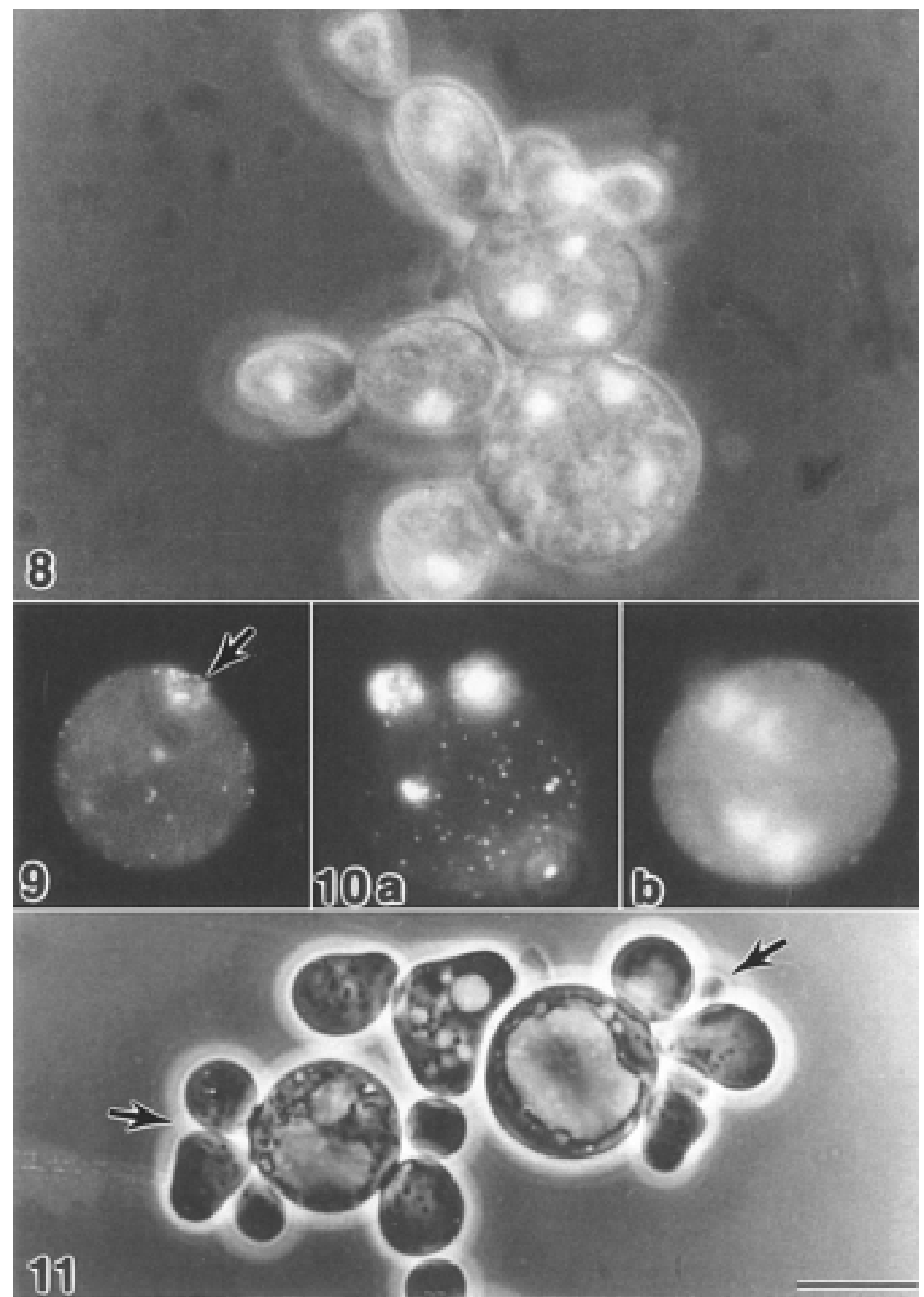

Figs 8-11. -8 . Morphology of a reverting protoplast. Note the decreasing size of the revertants. Phase contrast and DAPI staining. For Figs 8-11 the bar represents $10 \mu \mathrm{m}$. -9 . Cluster of subcortical actin patches before bud emergence (arrow). -10 . Three clusters of actin patches at the surface of a regenerated protoplast (a) and four nuclei in the cytoplasm (b). - 11. Axial pattern of budding of regenerated $71 \alpha \alpha$ protoplasts (arrows)

Acta Biologica Hungarica 52, 2001 
Buds on the protoplast surface were located in a random fashion which did not correspond to the bipolar distribution present in normal diploid or tetraploid cells. Reverting protoplasts of the diploid $71 \alpha \alpha$ cells also budded randomly, although some of them exhibited a cluster of buds at one site of the surface, which is a typical axial pattern of budding (Fig. 11).

\section{DISCUSSION}

The roles of actin and microtubules in cell growth and division have been repeatedly demonstrated: on elimination of the genes coding for the synthesis or assembly of actin or on the application of specific inhibitors, the polar growth of cells is disturbed and cytokinesis is inhibited [25]. It appears that the absence of actin cables is compatible with growth and division unless the assembly of actin patches is hindered [16]. The depolymerisation of microtubules does not interfere with polar growth and does not prevent initiation of the mating process [5]. Thus, the microtubules do not play any role in the control of the polar growth of yeast cells [7,25]. Our results demonstrated that a collapse of the microtubular skeleton and a degradation of actin, which occurs during protoplasting, did not necessarily have fatal consequences for the cell. Removal of the cell wall, however, does not lead to microtubule degradation and actin patch delocalisation; the same happens on the mere transfer of cells to a hypertonic medium used for protoplast formation [23].

The microtubular system was restored during 30 to $60 \mathrm{~min}$ of incubation and its recovery was not dependent on the presence of a cell wall. The extent to which the actin cytoskeleton is restored during protoplast regeneration is not yet clear. The redistribution of the actin patches under the protoplast surface is certainly artificial and resembles the effects of other stress factors [23]. However, during cell wall regeneration, in either liquid or solid media, this localisation is probably important in terms of function because the cell wall is synthesised all over the protoplast surface and the positions of the actin patches are undoubtedly related to this process $[2,10$, 17]. The precise role of these cortical actin patches is not known; however, there are indications that they are somehow involved in the final steps of secretion or endocytic recycling [22].

It was interesting to note that, even though the nuclei divided, the budding process was not triggered. This situation resembles the phenotypes of some $c d c$ mutants [1, $3,11]$. The fact that a multinucleate state in protoplasts is due to the absence of a complete cell wall could provide a clue to the deciphering mechanisms leading to a decoupling of karyokinesis from cytokinesis observed in some $c d c$ mutations.

The aggregation of the actin patches which precedes bud development takes place only after a structurally complete cell wall has been regenerated on the protoplast surface. The degree of completeness needed to trigger the budding process is not known. Electron microscopy studies have shown that the network of glucan fibrils is well cemented with amorphous material [15] and this layer is able to provide protoplasts with osmotic resistance. The aggregation of the actin patches acts as a signal 
that the whole multiprotein complex has been assembled and that the budding process can be triggered [7]. The time relation between the assembly of the budding complex and the completion of the cell wall suggests that the cell wall, or rather proteins interacting with it, may be one of the components which trigger budding and polar growth. It can be expected that there is a functional link between the cytoplasm and the cell wall, similarly to the link between the extracellular matrix and the cytoplasm in animal cells [12]. Integrins or integrin-like proteins involved in the molecular cross-talk between the extracellular matrix and cytoplasm have been described in some fungal cells $[6,8]$. In $S$. cerevisiae, a product of the BUD10 gene seems to be a candidate for signal transduction from the cytoplasm to the wall [7]. A search for the linkers that operate in the interface between the cytoplasm and the cell wall in yeast can be expected to yield interesting results.

\section{ACKNOWLEDGEMENTS}

This work was supported by grants No. 204 99/D025 and No. 204/00/0394 from the Grant Agency of the Czech Republic. The authors thank M. Zacharníková for her excellent technical assistance.

\section{REFERENCES}

1. Adams, A. E., Johnson, D. I., Longnecker, R. M., Sloat, B. F., Pringle, J. R. (1990) CDC42 and CDC43, two additional genes involved in budding and the establishment of cell polarity in the yeast Saccharomyces cerevisiae. J. Cell Biol. 111, 131-142.

2. Gabriel, M., Kopecká, M., Svoboda, A. (1992) Structural arrangement of the actin cytoskeleton in regenerating protoplasts of budding yeast. J. Gen. Microbiol. 138, 2229-2234.

3. Grallert, A., Grallert, B., Ribar, B., Sipiczki, M. (1998) Coordination of initiation of nuclear division and initiation of cell division in Schizosaccharomyces pombe: Genetic interactions of mutations. $J$. Bacteriol. 180, 892-900.

4. Hagan, I. M., Hyams, J. S. (1988) The use of cell division cycle mutants to investigate the control of microtubule distribution in the fission yeast Schizosaccharomyces pombe. J. Cell Sci. 89, 343-357.

5. Hašek, J., Rupeš, I., Svobodová, J., Streiblová. E. (1987) Tubulin and actin topology during zygote formation of Saccharomyces cerevisiae. J. Gen. Microbiol. 133, 3355-3363.

6. Hostetter, M. K. (1999) Integrin-like proteins in Candida spp. and other microorganisms. Fungal Genet. Biol. 28, 135-145.

7. Chant, J. (1999) Cell polarity in yeast. Annu. Rev. Cell Dev. Biol. 15, 365-391.

8. Kaminskyj, S. G. W., Heath, I. B. (1985) Integrin and spectrin homologues, and cytoplasm-wall adhesion in tip growth. J. Cell Sci. 108, 849-856.

9. Kobori, H., Yamada, N., Taki, A., Osumi, M. (1989) Actin is associated with the formation of the cell wall in reverting protoplasts of the fission yeast Schizosaccharomyces pombe. J. Cell Sci. 94, 635-646.

10. Kopecká, M., Gabriel, M. (1995) Actin cortical cytoskeleton and cell wall synthesis in regenerating protoplasts of the Saccharomyces cerevisiae actin mutant DBY 1693. Microbiology-UK 141, $1289-1299$

11. Lew, D. J., Reed, S. I. (1995) A cell cycle checkpoint monitors cell morphogenesis in budding yeast. J. Cell Biol. 129, 739-749.

12. Macieira-Coelho, A. (ed.) (2000) Signaling Through the Cell Matrix. Berlin, Springer-Verlag, p. 136.

13. Nečas, O., Svoboda, A. (1981) Morphogenesis in protoplasts. In Yeast Cell Envelopes: Biochemistry, Biophysics and Ultrastructure. W. N. Arnold (ed.) Boca Raton: CRC Press Inc., Vol. 2, pp. 105-128. 
14. Nečas, O., Svoboda, A. (1985) Cell wall regeneration and protoplast reversion. In Peberdy, J. F., Ferenczy, L. (eds). Fungal Protoplasts. New York, Marcel Dekker Inc., pp. 115-133.

15. Nečas, O. (1965) Mechanism of regeneration of yeast protoplasts. II. Formation of cell wall de novo. Folia Biol. (Praha) 11, 97-102.

16. Novick, P., Botstein, D. (1985) Phenotypic analysis of temperature-sensitive yeast actin mutants. Cell 40, 405-416.

17. Osumi, M., Sato, M., Ishijima, S. A., Konomi, M., Takagi, T., Yaguchi, H. (1998) Dynamics of cell wall formation in fission yeast, Schizosaccharomyces pombe. Fungal Genet. Biol. 24, 178-206.

18. Pokorná, I., Svoboda, A. (1995) Response of yeast protoplasts to their mating partners. Folia Microbiol. 40, 583-587.

19. Pringle, J. R., Adams, A. E., Drubin, D. G., Haarer, B. K. (1991) Immunofluorescence methods for yeast. Methods Enzymol. 194, 565-602.

20. Pringle, J. R., Preston, R. A., Adams, A. E., Stearns, T., Drubin, D. G., Haarer, B. K., Jones, E. W. (1989) Fluorescence microscopy methods for yeast. In Prescott, D. M. (ed). Methods in Cell Biology. New York, Academic Press, pp. 357-434.

21. Pruyne, D., Bretscher, A. (2000) Polarization of cell growth in yeast I. Establishment and maintenance of polarity states. J. Cell Sci. 113, 365-375.

22. Pruyne, D., Bretscher, A. (2000) Polarization of cell growth in yeast II. The role of the cortical actin cytoskeleton. J. Cell Sci. 113, 571-585.

23. Slaninová, I., Šesták, S., Svoboda, A., Farkaš, V. (2000) Cell wall and cytoskeleton reorganization as the response to hyperosmotic shock in Saccharomyces cerevisiae. Arch. Microbiol. 173, $245-252$.

24. Svoboda, A., Bähler, J., Kohli, J. (1995) Microtubule-driven nuclear movements and linear elements as meiosis-specific characteristics of the fission yeasts Schizosaccharomyces versatilis and Schizosaccharomyces pombe. Chromosoma 104, 203-214.

25. Winsor, B., Schiebel, E. (1997) Review: An overview of the Saccharomyces cerevisiae microtubule and microfilament cytoskeleton. Yeast 13, 399-434.

26. Woods, A., Sherwin, T., Sasse, R., MacRae, T. H., Baines, A. J., Gull, K. (1989) Definition of individual components within the cytoskeleton of Trypanosoma brucei by a library of monoclonal antibodies. J. Cell Sci. 93, 491-500. 
